\title{
On the closed subspaces of universal series in Banach spaces and Fréchet spaces
}

\author{
by \\ Stéphane Charpentier (Talence)
}

\begin{abstract}
We prove, in a general framework, the existence of a closed infinitedimensional subspace consisting of universal series.
\end{abstract}

Introduction. The study of universal series began in 1914, when Fekete showed that there exists a Taylor series on $[-1,1]$ whose subsequences of partial sums approximate uniformly any continuous function $f$ on $[-1,1]$ such that $f(0)=0$. The main idea of this result is that whatever can be uniformly approximated by polynomials can also be approximated by subsequences of partial sums of a Taylor series. After this, a lot of examples of universal series appeared in different spaces: Men'shov showed, for instance, the existence of many trigonometric series which approximate almost everywhere, along subsequences of their partial sums, any complex measurable $2 \pi$-periodic function on the torus. Other pointwise universality results were obtained concerning Faber series, Laurent series, Jacobi series, harmonic expansions, etc. For such results, we refer to the two surveys [6] and [7].

It turns out that, when a universal series does exist, the set of universal series has some nice properties. This was developed in [5] (in a more general context) and later in [2] and [14], where an abstract theory of universal series is presented. Precisely, it is shown there that, as soon as there exists a universal series, then the set of universal series is both generic (it contains a dense $G_{\delta}$ set) and algebraically generic (it contains a dense subspace, without 0 ). This is done in a general framework which covers every known example.

Independently, Bayart studied in [1] the existence of a closed infinitedimensional subspace of universal series. His work just deals with one particular example, that of universal Taylor series of holomorphic functions on the unit disk $D$. Our aim, in this paper, is to combine the ideas of [1] and [2] 
in order to show that, in the abstract framework of [2], we can always find a closed infinite-dimensional subspace (without 0) of universal series, as soon as one universal series exists.

This topic is not new in the context of universality. Indeed, a similar study was done for the so-called universal sequences of operators $T_{n}: X \rightarrow Y$ (see [4] and [12]). However, the sufficient condition of [12] is not helpful here (see Remark 2.3 below). It should be added that, in our context, no extra assumption for the existence of a closed infinite-dimensional subspace of universal series is needed.

The paper is organized as follows. The first section is devoted to the presentation of all concepts and results involved in this work, the second section presents the proof of the existence of a closed infinite-dimensional subspace, with 0 removed, of universal series in the most simple general case, namely when we deal with Banach spaces. In the third section, we partly generalize this result to the case of Fréchet spaces; in the fourth section, we are led to add some extra parameters, as the "center of expansion" of a universal series, in order to be able to manage most of the examples. In the last section, we give a brief glimpse of how our results can be illustrated by usual examples of universality.

1. Framework and results. Let $\left(X, d_{X}\right)$ be a metrizable vector space and let $\left(x_{n}\right)_{n \in \mathbb{N}}$ be a sequence in $X$. We suppose in the whole paper that $d_{X}$ is translation-invariant and that the topology of $X$ is defined by a countable family of seminorms denoted by $\left(\|\cdot\|_{n}\right)_{n \geq 0}$. Let also $A \subset \mathbb{k}^{\mathbb{N}}, \mathbb{k}=\mathbb{R}$ or $\mathbb{C}$, be a Fréchet space and $\left(e_{i}\right)_{i} \subset \mathbb{k}^{\mathbb{N}}$ be the sequence in $\mathbb{k}^{\mathbb{N}}$ defined by $e_{i}=$ $(0, \ldots, 0,1,0, \ldots)$ where 1 is at the $i$ th position. We denote by $d_{A}$ the distance in $A$ which makes it a Fréchet space and consequently $d_{A}$ is translationinvariant. We assume that $A$ satisfies the following conditions:

(1) $A$ contains the polynomials in $\mathbb{k}^{\mathbb{N}}$, that is, the elements which can be written as finite linear combinations of the $e_{i}$ 's with coefficients in $\mathbb{k}$.

(2) The set of polynomials in $\mathbb{k}^{\mathbb{N}}$, denoted by $G$, is dense in $A$.

(3) The coordinate projections

$$
p_{i}: A \rightarrow \mathbb{k}, \quad\left(a_{n}\right)_{n} \mapsto a_{i},
$$

are continuous for any $i \in \mathbb{N}$.

We will need the notions of valuation and degree of a polynomial in $\mathbb{k}^{\mathbb{N}}$ :

Definition 1.1. The valuation of a polynomial $P \in \mathbb{k}^{\mathbb{N}}$ is the index of its first non-zero coefficient, and its degree is the index of its last non-zero coefficient; we denote them respectively by $v(P)$ and $d(P)$.

We define the notion of unrestricted universality for an element of $X$, with respect to the sequence $\left(x_{n}\right)_{n}$, as follows: 
DeFinition 1.2. We say that $a=\left(a_{n}\right)_{n} \in \mathbb{k}^{\mathbb{N}}$ is unrestrictively universal (with respect to $\left.\left(x_{n}\right)_{n}\right)$ if the family $\left(\sum_{n=0}^{N} a_{n} x_{n}\right)_{N}$ is dense in $X$. We denote by $U$ the set of unrestricted universal sequences.

REMARK 1.1. Notice that if $U$ is non-empty, then $X$ is necessarily separable. We can even observe that there exists a dense subsequence in $X$ of vectors of the form $\sum_{n=0}^{N} a_{n} x_{n}$. These vectors are called polynomials in $X$ with respect to $\left(x_{n}\right)_{n}$.

This remark leads us to denote by the same letter a polynomial $\left(a_{n}\right)_{n}$ in $\mathbb{K}^{\mathbb{N}}$ and the polynomial $\sum_{n} a_{n} x_{n}$ in $X$, if no confusion can arise. If $a=$ $\left(a_{n}\right)_{n}$ is more generally an element of $A$, we will denote by $S_{N}(a)$ the partial sum of order $N, \sum_{n=0}^{N} a_{n} e_{n}$ in $A$, and we will distinguish it from $S_{N}(a, X)=$ $\sum_{n=0}^{N} a_{n} x_{n}$, the partial sum of $a$ in $X$ with respect to $\left(x_{n}\right)_{n}$.

In this paper, we will also be interested in the notion of restricted universality:

Definition 1.3. An element $a=\left(a_{n}\right)_{n} \in A$ is said to be restrictively universal (with respect to $\left(x_{n}\right)_{n}$ ) if, for every $x \in X$, there exists an increasing sequence $\left(\lambda_{N}\right)_{N \in \mathbb{N}}$ of integers such that

(1) $\sum_{n=0}^{\lambda_{N}} a_{n} x_{n}$ converges to $x$ when $N$ goes to infinity,

(2) $\sum_{n=0}^{\lambda_{N}} a_{n} e_{n}$ converges to $a$ when $N$ goes to infinity.

We denote by $U_{A}$ the set of restricted universal sequences.

Of course, every restricted universal sequence is unrestricted, and we notice immediately that if $\left(e_{n}\right)_{n}$ is a Schauder basis of $A$, then $U \cap A=U_{A}$; so that the notion of restricted universal sequences has an interest when $\left(e_{n}\right)_{n}$ is not a Schauder basis of $A$.

Our result is based on the following one, given in [2], which characterizes the non-emptiness of the set $U_{A}$.

THEOREM 1.1. Under the previous assumptions, the following are equivalent:

(1) $U_{A}$ is non-empty.

(2) For every $\varepsilon>0$, every $x \in X$, and every integer $p \geq 0$, there exists a polynomial $\left(a_{n}\right)_{n}$ with valuation $p$ such that

$$
d_{X}\left(\sum_{n \in \mathbb{N}} a_{n} x_{n}, x\right)<\varepsilon \quad \text { and } \quad d_{A}\left(\sum_{n \in \mathbb{N}} a_{n} e_{n}, 0\right)<\varepsilon .
$$

(3) $U_{A} \cup\{0\}$ is a dense $G_{\delta}$ subset of $A$ and it contains a dense subspace of $A$.

Our aim in this paper is to show that when $U_{A}$ is non-empty, it contains automatically a closed infinite-dimensional subspace of $A$ without 0 . We will 
first give a proof when $A$ is a Banach space; this proof will be partly based on the possibility of building a convenient basic sequence of the Banach space $A$. This trick fails when $A$ is a Fréchet space. Thus, in the latter case, we just show that $U \cap A$ (and not $U_{A}$ ) contains a closed infinite-dimensional subspace of $A$ without 0 . However, in all concrete examples, $U_{A}$ is equal to $U \cap A$. Therefore, we can say that the problem is solved in the main cases.

2. Universality in Banach spaces. In this section, we deal with the case where $A$ is a Banach space, with a norm $\|\cdot\|_{A}$. For this, we will need to show how to build a normalized basic sequence in $A$ whose elements can be chosen as polynomials with an arbitrary valuation. This is the purpose of the following lemma:

Lemma 2.1. Let $\varepsilon>0$, and let $\left(u_{0}, \ldots, u_{n}\right) \subseteq A$. For every integer $v$, there exists a polynomial $u_{n+1} \in A$ such that $v\left(u_{n+1}\right) \geq v,\left\|u_{n+1}\right\|_{A}=1$ and

$$
\left\|\sum_{k=0}^{n} \lambda_{k} u_{k}\right\|_{A} \leq(1+\varepsilon)\left\|\sum_{k=0}^{n} \lambda_{k} u_{k}+\lambda u_{n+1}\right\|_{A}
$$

for any scalars $\lambda$ and $\lambda_{k}, 0 \leq k \leq n$.

REMARK 2.1. If $\left(\varepsilon_{n}\right)_{n \geq 0}$ is a sequence of positive real numbers such that $\prod_{n=0}^{\infty}\left(1+\varepsilon_{n}\right)$ converges to $K$, then defining for example $u_{0}=e_{0} /\left\|e_{0}\right\|_{A}$, we can construct by induction a basic sequence $\left(u_{n}\right)_{n \geq 0}$ with basis constant not greater than $K$, such that every term is built as in the previous lemma, by taking $\varepsilon=\varepsilon_{n}$ at step $n$.

Indeed, for every integer $p$ and $q$ with $q \geq p$, Lemma 2.1 allows us to construct polynomials $u_{k}, 0 \leq k \leq q$, such that, for any scalars $\left(\lambda_{k}\right)_{0 \leq k \leq q}$,

$$
\left\|\sum_{k=0}^{p} \lambda_{k} u_{k}\right\|_{A} \leq \prod_{k=p}^{q-1}\left(1+\varepsilon_{k}\right)\left\|\sum_{k=0}^{q} \lambda_{k} u_{k}\right\|_{A} \leq K\left\|\sum_{k=0}^{q} \lambda_{k} u_{k}\right\|_{A} .
$$

Proof of Lemma 2.1. Let $\varepsilon>0$ and $\left(u_{0}, \ldots, u_{n}\right) \subseteq A$. Let also $v$ be an integer. Let $\left(z_{j}\right)_{j=0}^{l_{n+1}}$ be an $\frac{\varepsilon}{1+\varepsilon}$-net of the unit sphere of $\operatorname{span}\left(u_{0}, \ldots, u_{n}\right)$ and $\left(\varphi_{j}\right)_{j=0}^{l_{n+1}}$ be continuous linear functionals on $A$ of norm 1 such that, for every $0 \leq j \leq l_{n+1}$,

$$
\varphi_{j}\left(z_{j}\right)=1 .
$$

As $\operatorname{codim}\left(\left(\bigcap_{j=0}^{l_{n+1}} \operatorname{ker}\left(\varphi_{j}\right)\right) \cap\left(\bigcap_{i=0}^{v} \operatorname{ker}\left(e_{i}^{*}\right)\right)\right) \leq l_{n+1}+v+2$, there exists an integer $m_{n+1}$ such that, if we denote

$$
F_{n+1}=\operatorname{span}\left(e_{0}, \ldots, e_{m_{n+1}}\right),
$$


there exists $u_{n+1} \in F_{n+1}$ of norm 1 such that

$$
u_{n+1} \in\left(\bigcap_{j=0}^{l_{n+1}} \operatorname{ker}\left(\varphi_{j}\right)\right) \cap\left(\bigcap_{i=0}^{v} \operatorname{ker}\left(e_{i}^{*}\right)\right) .
$$

By construction, $u_{n+1}$ is a polynomial of valuation greater than $v$, and of norm 1. It just remains to verify that $u_{n+1}$ satisfies (2.1). Let $\lambda$ be an arbitrary scalar and let $j_{0}$ be an index such that

$$
\left\|\frac{\sum_{k=0}^{n} \lambda_{k} u_{k}}{\left\|\sum_{k=0}^{n} \lambda_{k} u_{k}\right\|_{A}}-z_{j_{0}}\right\|_{A} \leq \frac{\varepsilon}{1+\varepsilon} .
$$

By definition, we have

$$
\begin{aligned}
1 & =\varphi_{j_{0}}\left(z_{j_{0}}+\lambda u_{n+1}\right) \leq\left\|z_{j_{0}}+\lambda u_{n+1}\right\|_{A} \\
& \leq\left\|z_{j_{0}}-\frac{\sum_{k=0}^{n} \lambda_{k} u_{k}}{\left\|\sum_{k=0}^{n} \lambda_{k} u_{k}\right\|_{A}}\right\|_{A}+\left\|\frac{\sum_{k=0}^{n} \lambda_{k} u_{k}+\lambda\left\|\sum_{k=0}^{n} \lambda_{k} u_{k}\right\|_{A} u_{n+1}}{\left\|\sum_{k=0}^{n} \lambda_{k} u_{k}\right\|_{A}}\right\|_{A} \\
& \leq \frac{\varepsilon}{1+\varepsilon}+\left\|\frac{\sum_{k=0}^{n+1} \lambda_{k} u_{k}}{\left\|\sum_{k=0}^{n} \lambda_{k} u_{k}\right\|_{A}}\right\|_{A}, \quad \text { by denoting } \lambda_{n+1}=\lambda\left\|\sum_{k=0}^{n} \lambda_{k} u_{k}\right\|_{A} .
\end{aligned}
$$

Hence

$$
\left(1-\frac{\varepsilon}{1+\varepsilon}\right)\left\|\sum_{k=0}^{n} \lambda_{k} u_{k}\right\|_{A} \leq\left\|\sum_{k=0}^{n+1} \lambda_{k} u_{k}\right\|_{A},
$$

so

$$
\left\|\sum_{k=0}^{n} \lambda_{k} u_{k}\right\|_{A} \leq(1+\varepsilon)\left\|\sum_{k=0}^{n+1} \lambda_{k} u_{k}\right\|_{A} .
$$

Up to dividing $\lambda$ by $\left\|\sum_{k=0}^{n} \lambda_{k} u_{k}\right\|_{A}$, we obtain the result.

This lemma is just an improvement of the construction of a basic sequence in a Banach space, given by S. Mazur (see [10, Theorem 1.a.5, p. 4]). When Theorem 1.1 is the main tool to show that the set $U$ contains a closed infinite-dimensional subspace of $A$, this lemma allows us to replace $U$ by $U_{A}$.

THEOREM 2.1. Under the previous assumptions and notations, if $U_{A} \neq \emptyset$, then it contains a closed infinite-dimensional subspace of $A$ without 0.

Proof. We will build by induction simultaneously a family $\left(f_{n, k}\right)_{n \geq 0,0 \leq k \leq n}$ of elements of $A$ and a "convenient" basic sequence $\left(u_{k}\right)_{k}$ of $A$, thanks to which we will define a subspace as desired. For this purpose, let $\left(Q_{l}\right)_{l}$ be a sequence of polynomials dense in $X$ and let $\varphi, \psi: \mathbb{N} \rightarrow \mathbb{N}$ be two functions such that, given any $(m, t) \in \mathbb{N}^{2}$, there exists an infinite family $\left(v_{k}\right)_{k} \subseteq \mathbb{N}$ 
such that $\left(\varphi\left(v_{k}\right), \psi\left(v_{k}\right)\right)=(m, t)$ for every $k$. Let also $\left(\varepsilon_{n}\right)_{n \geq 0}$ be a sequence of positive real numbers such that $\prod_{n=0}^{\infty}\left(1+\varepsilon_{n}\right)$ converges to a real number $K$.

The order of the inductive construction of the $f_{n, k}$ 's will be the following: first, we define $f_{0,0}$ from $u_{0}$, then we build $f_{1,0}$ from $f_{0,0}$, then $f_{1,1}$ from $u_{1}$, then $f_{2,0}$ from $f_{1,0}, f_{2,1}$ from $f_{1,1}$ and so on. This order is important because we will take into account all the previous $f_{l, t}$ for the construction of $f_{n, k}$. Each $f_{n, k}$ will consist of a first part, $g_{n, k}$, which will approximate the polynomial $Q_{\varphi(n)}$ in $X$ and of a second part which will annihilate the value of $g_{n, k}$ in $A$. This second part will be necessary to ensure convergence of $\left(f_{n, k}\right)_{n}$ in $A$ and that its limit is close to $u_{k}$.

First, we fix $u_{0}=e_{0} /\left\|e_{0}\right\|_{A}$ and approximate $Q_{\varphi(0)}$ in $X$ by $g_{0,0}=u_{0}+P$, the polynomial in $\mathbb{k}^{\mathbb{N}}$ where $P$ is given by Theorem $1.1(2)$ applied to the spaces $\left(A, d_{A}\right)$ and $\left(X,\|\cdot\|_{\psi(0)}\right)$, with $x=Q_{\varphi(0)}-u_{0}, p=2$ and $\varepsilon=1 / 2^{4} K$. Next we put $f_{0,0}=g_{0,0}+Q$, where $Q$ is given by Theorem 1.1. (2) for $\left(A, d_{A}\right)$ and $\left(X,\|\cdot\|_{\psi(1)}\right)$ with $x=-g_{0,0}, p=d(P)+1$ and $\varepsilon=1 / 2^{4} K$. Then $g_{0,0}$ and $f_{0,0}$ satisfy the following conditions:

$$
\begin{aligned}
\left\|g_{0,0}-Q_{\varphi(0)}\right\|_{\psi(0)} & <\frac{1}{2^{4} K}, \\
\left\|f_{0,0}\right\|_{\psi(1)} & <\frac{1}{2^{4} K}, \\
\left\|f_{0,0}-u_{0}\right\|_{A} & <\frac{1}{2^{3} K} .
\end{aligned}
$$

Now, we turn to the inductive step. So, fix $n \in \mathbb{N}$ and suppose that polynomials $f_{j, l}$, for $l \leq j \leq n-1$ have been built. We denote by $\prec$ the lexicographical order on $\mathbb{N}^{2}$. We will construct the polynomials $f_{n, k}$ following the lexicographical order. First, we build $f_{n, k}$ for $k<n$ as follows.

We approximate the polynomial $Q_{\varphi(n)}$ in $X$ by setting $g_{n, k}=f_{n-1, k}+P$, where $P$ is given by Theorem 1.1 , applied to $\left(A, d_{A}\right)$ and $\left(X,\|\cdot\|_{\psi(n)}\right)$ with $x=Q_{\varphi(n)}-f_{n-1, k}, p=\max \left(d\left(f_{j, l}\right),(j, l) \prec(n, k)\right)+1$ and $\varepsilon=1 / 2^{n+4} K$. Then we set $f_{n, k}=g_{n, k}+Q$, where $Q$ is given by Theorem 1.1 for $\left(A, d_{A}\right)$ and $\left(X,\|\cdot\|_{\psi(n+1)}\right)$ with $x=-g_{n, k}, p=d(P)+1$ and $\varepsilon=1 / 2^{n+4} K$.

We finally build $u_{n}$ and $f_{n, n}$. We deduce $u_{n}$ from $u_{n-1}$ by induction and $f_{n, n}$ from $u_{n}$. We put $g_{n, n}=u_{n}+P$, with $u_{n}$ given by Lemma 2.1 with $B=\left(u_{1}, \ldots, u_{n-1}\right), v=p_{n}=\max \left(d\left(f_{j, l}\right),(j, l) \prec(n, n)\right)+1$ and $\varepsilon=\varepsilon_{n}$, and where $P$ comes from Theorem 1.1, applied to the spaces $\left(A, d_{A}\right)$ and $\left(X,\|\cdot\|_{\psi(n)}\right)$, where $x=Q_{\varphi(n)}-u_{n}, p=d\left(u_{n}\right)+1$ and $\varepsilon=1 / 2^{n+4} K$. Notice that as $u_{n}$ is a polynomial, the definition of $g_{n, n}$ makes sense in $X$. Then we define $f_{n, n}=g_{n, n}+Q$, where $Q$ is given by Theorem 1.1 for $\left(A, d_{A}\right)$ and $\left(X,\|\cdot\|_{\psi(n+1)}\right)$ with $x=-g_{n, n}, p=d(P)+1$ and $\varepsilon=1 / 2^{n+4} K$. As above, 
for $n \geq k, g_{n, k}, u_{k}$ and $f_{n, k}$ satisfy

$$
\begin{aligned}
\left\|g_{n, k}-Q_{\varphi(n)}\right\|_{\psi(n)} & <\frac{1}{2^{n+4} K}, \\
\left\|f_{n, k}\right\|_{\psi(n+1)} & <\frac{1}{2^{n+4} K}, \\
\left\|f_{n+1, k}-f_{n, k}\right\|_{A} & <\frac{1}{2^{n+4} K}, \\
\left\|f_{k, k}-u_{k}\right\|_{A} & <\frac{1}{2^{k+3} K},
\end{aligned}
$$

and $\left(u_{k}\right)_{k}$ is a basic sequence of $A$ with basis constant not greater than $K$, according to Remark 2.1.

Using inequality (2.4), we define for every $k \in \mathbb{N}$ an element $f_{k}$ of $A$ by

$$
f_{k}=\sum_{n=k}^{\infty}\left(f_{n+1, k}-f_{n, k}\right)+f_{k, k}=\lim _{n \rightarrow \infty} f_{n, k} .
$$

By construction, the family $\left(f_{k}\right)_{k}$ is linearly independent: indeed, as

$$
d\left(f_{n_{1}, k_{1}}\right)<v\left(f_{n_{2}, k_{2}}\right) \quad \text { for }\left(n_{1}, k_{1}\right) \prec\left(n_{2}, k_{2}\right)
$$

and as the linear coordinate projections $p_{i}$ (see the introduction) are supposed to be continuous, an identity like $\sum_{k=0}^{N} \alpha_{k} f_{k}=0$ for an integer $N$ can hold only if $\alpha_{k}=0$ for every $k$. Therefore, the subspace $E$ of $A$ defined by

$$
E=\left\{\sum_{k=0}^{\infty} \alpha_{k} f_{k}: \sum_{k=0}^{\infty} \alpha_{k} f_{k} \text { converges }\right\}
$$

is infinite-dimensional and we will verify that the closed infinite-dimensional subspace $F=\bar{E}$ answers our question, i.e. every non-zero element of $F$ is universal. First of all, observe that, in fact, every element $h$ of $F$ can be written as $\sum_{k=0}^{\infty} \alpha_{k} f_{k}$ with $\alpha_{k} \in \mathbb{k}$, so that $E$ is closed and $F=E$. Indeed, by construction and using inequalities (2.4) and (2.5), for any $k$ we have

$$
\begin{aligned}
\left\|f_{k}-u_{k}\right\|_{A} & =\left\|\sum_{n=k}^{\infty}\left(f_{n+1, k}-f_{n, k}\right)+f_{k, k}-u_{k}\right\| \\
& <\sum_{n=k}^{\infty} \frac{1}{2^{n+4} K}+\frac{1}{2^{k+3} K}<\frac{1}{2^{k+2} K} .
\end{aligned}
$$

Moreover, as $\left(u_{k}\right)_{k}$ is a basic sequence with $A$ with basis constant not greater than $K, u_{k}^{*}$ is well-defined and $\left\|u_{k}^{*}\right\|_{A^{*}}\left\|u_{k}\right\|_{A} \leq 2 K$; by construction, $\left\|u_{k}\right\|_{A}=1$ for any $k$; therefore $(2.6)$ gives us

$$
\sum_{k=0}^{\infty}\left\|u_{k}^{*}\right\|_{A^{*}}\left\|f_{k}-u_{k}\right\|_{A}<\sum_{k=0}^{\infty} \frac{1}{2^{k+1}}=1 .
$$

Then the Bessaga-Pełczyński criterion ensures that $\left(f_{k}\right)_{k}$ is a basic sequence of $A$ equivalent to $\left(u_{k}\right)_{k}$ so that $E=\bar{E}=F$. 
Now we take a (non-zero) element $h=\sum_{k=0}^{\infty} \alpha_{k} f_{k} \in E=F$ and we show that $h$ is universal. Given a polynomial $Q_{l}$ of the enumeration of polynomials dense in $A$, it is sufficient to prove that there exists a sequence $N_{j}$ (depending on $h$ and $Q_{l}$ ) of integers such that

$$
S_{N_{j}}(h, X) \underset{j \rightarrow \infty}{\longrightarrow} Q_{l} \quad \text { for the topology of }\left(X, d_{X}\right),
$$

and for this it is sufficient to prove that, for every $n \in \mathbb{N}$,

$$
\left\|S_{N_{j}}(h, X)-Q_{l}\right\|_{n} \underset{n \rightarrow \infty}{\longrightarrow} 0 .
$$

We denote by $k_{0}$ the smallest integer $k$ such that $\alpha_{k} \neq 0$ and by $N_{j}$ the degree of the polynomial $g_{v_{j}+k_{0}, k_{0}}$. By multiplying each $\alpha_{k}$ by $\left(\alpha_{k_{0}}\right)^{-1}$, we may suppose that $\alpha_{k_{0}}=1$. By definition of $\varphi$ and $\psi$, there exists an increasing sequence $\left(v_{j}\right)_{j}$ such that $\left(\varphi\left(v_{j}+k_{0}\right), \psi\left(v_{j}+k_{0}\right)\right)=(l, n)$ for every $j$. Then

$$
\begin{aligned}
\left\|S_{N_{j}}(h, X)-Q_{l}\right\|_{n}=\left\|S_{N_{j}}\left(\sum_{k=k_{0}}^{v_{j}+k_{0}} \alpha_{k} f_{k}, X\right)-Q_{l}\right\|_{\psi\left(v_{j}+k_{0}\right)} \\
=\left\|\sum_{k=k_{0}}^{v_{j}-1+k_{0}} \alpha_{k} f_{v_{j}-1+k_{0}, k}+g_{v_{j}+k_{0}, k_{0}}-Q_{l}\right\|_{\psi\left(v_{j}+k_{0}\right)} \\
\leq\left\|\sum_{k=k_{0}}^{v_{j}-1+k_{0}} \alpha_{k} f_{v_{j}-1+k_{0}, k}\right\|_{\psi\left(v_{j}+k_{0}\right)}+\left\|g_{v_{j}+k_{0}, k_{0}}-Q_{l}\right\|_{\psi\left(v_{j}+k_{0}\right)} \\
\leq\left\|\sum_{k=k_{0}}^{v_{j}-1+k_{0}} \alpha_{k} f_{v_{j}-1+k_{0}, k}\right\|_{\psi\left(v_{j}+k_{0}\right)}+\frac{1}{2^{v_{j}+k_{0}+4} K},
\end{aligned}
$$

using (2.2). Moreover, using (2.6), and the fact that $\left\|u_{k}\right\|_{A}=1$ for any $k$, we get

$$
\left|\alpha_{k}\right| \leq \frac{2 K^{\prime}}{\left\|f_{k}\right\|_{A}}
$$

with $\left\|f_{k}\right\|_{A} \geq 1-1 / 2^{k+3} K^{\prime}$ for every $k$, where $K^{\prime}$ is the basis constant of $\left(f_{k}\right)_{k}$. Thus the sequence $\left(\alpha_{k}\right)_{k}$ is bounded, say by $M ; 2.7$ entails that

$$
\begin{aligned}
\left\|S_{N_{j}}(h, X)-Q_{l}\right\|_{n} & \leq M \sum_{k=k_{0}}^{v_{j}-1+k_{0}}\left\|f_{v_{j}-1+k_{0}, k}\right\|_{\psi\left(v_{j}+k_{0}\right)}+\frac{1}{2^{v_{j}+k_{0}+4} K} \\
& \leq \frac{M\left(v_{j}-1\right)}{2^{v_{j}+3+k_{0}} K}+\frac{1}{2^{v_{j}+k_{0}+4} K} \quad \text { by } 2.3 \\
& \rightarrow 0 \quad \text { as } j \rightarrow \infty \text { since }\left(v_{j}\right)_{j} \text { is increasing, }
\end{aligned}
$$

which proves the universality of $h$.

The proof of the theorem will be finished after verifying that $S_{N_{j}}(h)$ converges to $h$ in $A$. This easily comes from the construction of the $f_{k}$ 's by 
using the triangle inequality. Indeed, we have

$$
\begin{aligned}
\left\|h-S_{N_{j}}(h)\right\|_{A} \leq & \left\|\sum_{k \geq v_{j}+k_{0}+1} \alpha_{k} f_{k}\right\|_{A} \\
& +\left\|\sum_{k=k_{0}}^{v_{j}+k_{0}} \alpha_{k}\left(\sum_{n \geq v_{j}+k_{0}+1}\left(f_{n+1, k}-f_{n, k}\right)\right)\right\|_{A} \\
& +\left\|\alpha_{v_{j}+k_{0}}\left(f_{v_{j}+k_{0}, v_{j}+k_{0}}-g_{v_{j}+k_{0}, v_{j}+k_{0}}\right)\right\|_{A} \\
\leq & \left\|\sum_{k \geq v_{j}+k_{0}+1} \alpha_{k} f_{k}\right\|_{A}+\sum_{k=k_{0}}^{v_{j}+k_{0}}\left|\alpha_{k}\right|\left(\sum_{n \geq v_{j}+k_{0}+1} \frac{1}{2^{n+4} K}\right) \\
& +\left|\alpha_{v_{j}+k_{0}}\right| \frac{1}{2^{v_{j}+k_{0}+4} K} \\
\leq & \left\|\sum_{k \geq v_{j}+k_{0}+1} \alpha_{k} f_{k}\right\|_{A}+\frac{1}{16 K} \sup _{k \geq k_{0}}\left|\alpha_{k}\right| \sum_{k=k_{0}}^{v_{j}+k_{0}} \frac{1}{2^{v_{j}+k_{0}}} \\
& +\left|\alpha_{v_{j}+k_{0}}\right| \frac{1}{2^{v_{j}+k_{0}+4} K} \\
\rightarrow & 0 \quad \text { as } j \rightarrow \infty,
\end{aligned}
$$

since the series $\sum_{k \geq 0} \alpha_{k} f_{k}$ is convergent and the sequence $\left(\alpha_{k}\right)_{k}$ is bounded, which gives the result.

REMARK 2.2. Notice that we only use implication $(1) \Rightarrow(2)$ of Theorem 1.1. which does not require the continuity of the projections $p_{i}$ 's. In Theorem 1.1. this hypothesis is made to ensure that $U_{A}$ is "large"; this is also the case in the last proof: continuity of the $p_{i}$ 's is crucial to show that the subspace $E$ which we construct is infinite-dimensional.

The calculation which allows us to show that an element of $E$ is universal is the main reason to assume that $X$ has a topology induced by a family of seminorms. If this is not the case, our method is not successful, because the lack of homogeneity of the distance does not allow us to place the coefficients $\alpha_{k}$ in front of the distance and use the fact that they are uniformly bounded.

REMARK 2.3. More generally, let $\left(T_{n}\right)_{n}$ be a sequence of operators from $X$ to $Y$ where $X$ and $Y$ are two Banach spaces. A vector $x \in X$ is called universal for $\left(T_{n}\right)$ if the set $\left\{T_{n} x: n \in \mathbb{N}\right\}$ is dense in $Y$. A sufficient condition for the set of universal vectors to contain a closed infinite-dimensional subspace is given for instance in [12]. This condition requires that $\left(T_{n}\right)$ satisfies the so-called Universality Criterion (see for instance [3]). In our context, the operator $\left(T_{N}\right)$ is simply the function which maps $\left(a_{n}\right) \in A$ to the partial sum $S_{N}(a)=\sum_{n=0}^{N} a_{n} x_{n}$ in $X$. There is no reason why this sequence of 
maps should satisfy, in general, the Universality Criterion. Thus, the result of [12] does not cover our Theorem 2.1.

Theorem 2.1 can be almost generalized to the case of Fréchet spaces; that is the purpose of the forthcoming section.

3. Universality in Fréchet spaces. We keep the notations of the introduction, and for instance $A$ is now a Fréchet subspace of $\mathbb{K}^{\mathbb{N}}$ with a translation-invariant metric $d_{A}$; we also suppose that there is a norm $\widehat{\|\cdot\|}$ over $\left(A, d_{A}\right)$ such that there exists a constant $C>0$ satisfying

$$
\widehat{\|x\|} \leq C d_{A}(x, 0), \quad \forall x \in A .
$$

This condition, which means that $A$ admits a continuous norm, is of course equivalent to the fact that the topology of $A$ is generated by a family of norms. This assumption is often made in similar contexts. For example, this is the case in Theorem 3.1 of [4] where the authors give a sufficient condition for some sets of universal vectors to contain a closed infinite-dimensional subspace (see Remark 2.3). They exhibit a counterexample when this assumption is removed ([4, Example 3.2]). Moreover, Fréchet spaces of complex sequences which can be identified to spaces of holomorphic functions in one variable are typical spaces which satisfy (3.1).

In the present case, we do not have a result as good as the one obtained in Theorem 2.1 for Banach spaces: we will show that $\left(A, d_{A}\right)$ contains a closed infinite-dimensional subspace whose non-zero elements are all universal (with respect to $X$ ), so that $U \cup\{0\}$ contains a closed infinite-dimensional subspace of $A$; but our methods fail to show that it is also the case of $U_{A} \cup\{0\}$, unless of course we suppose that $\left(e_{n}\right)_{n}$ is a basis of $A$, i.e. $U_{A}=U$, which nevertheless covers most of the reasonable cases (including spaces of holomorphic functions in one variable). We state our result as follows:

THEOREM 3.1. With the previous notations, if $U_{A}$ is non-empty, then $U$ contains a closed infinite-dimensional subspace of $A$ without 0 .

Proof. The hypothesis (3.1) will permit us to treat Fréchet spaces almost as if they were Banach spaces from the point of view of basic sequences. In fact, we consider the Banach space $(\widehat{A}, \widehat{\|\cdot\|})$ obtained by completing $A$ for the norm $\widehat{\|\cdot\|}$ which satisfies 3.1 . This idea was inspired by the second part of the proof of Theorem 2 in [1]. Let $\left(Q_{l}\right)_{l \geq 1}$ be an enumeration of a dense family of polynomials of $X$ and let $\varphi, \psi$ be two functions from $\mathbb{N}$ to $\mathbb{N}$ such that for every couple of integers $(l, t)$, there exist infinitely many integers $j$ such that $(\varphi(j), \psi(j))=(l, t)$. By using Theorem 1.1. we simultaneously construct, as in the proof of Theorem 2.1 and with the same notations, three families $\left(\left(f_{n, k}\right)_{n \geq k}\right)_{k \geq 0},\left(\left(g_{n, k}\right)_{n \geq k}\right)_{k \geq 0}$ and $\left(u_{n}\right)_{n \geq 0}$ of poly- 
nomials in $A$ (or $X$, with no confusion possible), with convenient valuation and degree, such that

$$
\begin{aligned}
\left\|g_{n, k}-Q_{\varphi(n)}\right\|_{\psi(n)} & <\frac{1}{2^{n+4} K}, \\
\left\|f_{n, k}\right\|_{\psi(n+1)} & <\frac{1}{2^{n+4} K}, \\
\left\|f_{n+1, k}-f_{n, k}\right\| \leq C d_{A}\left(f_{n+1, k}, f_{n, k}\right) & <\frac{1}{2^{n+4} K}, \\
\left\|\widehat{f_{k, k}-u_{k}}\right\| \leq C d_{A}\left(f_{k, k}, u_{k}\right) & <\frac{1}{2^{n+3} K},
\end{aligned}
$$

where the constructions of $\left(\left(f_{n, k}\right)_{n \geq k}\right)_{k \geq 0}$ and $\left(\left(g_{n, k}\right)_{n \geq k}\right)_{k \geq 0}$ are done in $\left(A, d_{A}\right)$ and that of the basic sequence $\left(u_{n}\right)_{n \geq 1}$ of the latter space in $(\widehat{A}, \widehat{\|\cdot\|})$. It is important to notice that Theorem 1.1 is only applied to $\left(A, d_{A}\right)$ and not to the completion $(\widehat{A}, \widehat{\|\cdot\|})$, even for the construction of $f_{k, k}$ and to obtain inequality 3.5 . Indeed, the $u_{n}$ 's are built in $(\widehat{A}, \widehat{\|\cdot\|})$ as polynomials, and so they belong to $\left(A, d_{A}\right)$ so that we can use Theorem 1.1 to $\left(A, d_{A}\right)$ in order to deduce the construction of $f_{k, k}$ in $\left(A, d_{A}\right)$ from $u_{k}$. Moreover, this process consisting in passing from $\left(A, d_{A}\right)$ to $(\widehat{A}, \widehat{\|\cdot\|})$ is allowed by assumption $(3.1)$. We then define the $f_{k}$ 's in $\left(A, d_{A}\right)$ by

$$
f_{k}=\sum_{n \geq k}\left(f_{n+1, k}-f_{n, k}\right)+f_{k, k}
$$

and we consider the closed infinite-dimensional subspace $F$ of $\left(A, d_{A}\right)$ defined by

$$
F=\bar{E}^{d_{A}}
$$

where $E=\left\{\sum_{k>0} \alpha_{k} f_{k}\right.$ : the series converges in $\left.\left(A, d_{A}\right), \alpha_{k} \in \mathbb{k}\right\}$ and $\bar{E}^{d_{A}}$ is the closure of $E$ in $\left(A, d_{A}\right)$. Notice that the convergence in $\left(A, d_{A}\right)$ implies the convergence in $(\widehat{A}, \widehat{\|\cdot\|})$ according to $(3.1)$, hence $F \subseteq(\widehat{A}, \widehat{\|\cdot\|})$. To finish the proof, it is sufficient to verify that every non-zero element of $F$, seen as an element of $(\widehat{A}, \widehat{\|\cdot\|})$, is universal (we are now working in $X$ ). For this, we consider the set $\widetilde{E}=\left\{\sum_{k \geq 0} \alpha_{k} f_{k}\right.$ : the series converges in $\left.(\widehat{A}, \widehat{\|\cdot\|}), \alpha_{k} \in \mathbb{k}\right\}$ and we notice that $F \subseteq \widetilde{E}$. Now, we obtain the universality of every non-zero element of $\widetilde{E}$ directly from hypothesis 3.1 , inequalities $3.2-3.5$, and the proof of Theorem 2.1. by using the fact that the sequence $\left(u_{n}\right)_{n}$ is basic and normalized in the Banach space $(\widehat{A}, \widehat{\|\cdot\|})$ and therefore $\left(f_{k}\right)_{k}$ is also a basic sequence in $(\widehat{A}, \widehat{\|\cdot\|})$.

Let us recall

THEOREM (Theorem 30 of [2]). Under the previous assumptions, the following are equivalent: 
(1) $U \cap A$ is non-empty.

(2) $U \cap A$ is a dense $G_{\delta}$ subset of $A$.

(3) For every $\varepsilon>0$ and every $x \in X$, there exist $m, n \in \mathbb{N}, m \geq n$, and $a_{0}, a_{1}, \ldots, a_{m}$ in $\mathbb{k}$ such that

$$
d_{X}\left(\sum_{j=0}^{n} a_{j} x_{j}, x\right)<\varepsilon \quad \text { and } \quad d_{A}\left(\sum_{j=0}^{m} a_{j} e_{j}, 0\right)<\varepsilon .
$$

Looking at this result, our Theorem 3.1 may be a little disappointing. Indeed, comparing our results in Banach spaces and in Fréchet spaces, we can wonder if assuming $U_{A} \neq \emptyset$ in the second case is not too strong, since we just infer that $U \cap A$ contains a closed infinite-dimensional subspace. At this point, Theorem 30 of [2] can appear as the one to appeal to in the Fréchet case. So a natural question can be: can we replace the hypothesis $U_{A} \neq \emptyset$ by $U \cap A \neq \emptyset$ in Theorem 3.1? However, for technical reasons (like the appearance of the two indices $n$ and $m$ that we cannot control), it turns out that it is not so evident that we can apply Theorem 30 of [2] in order to positively answer the last question. Moreover, notice that we do not know if $U \cap A$ contains a dense subspace of $A$ under the assumption $U \cap A \neq \emptyset$. In fact, it is not even clear that it contains a subspace of dimension 2.

4. Labeled universal series. In the theory of universal series, we may deal with the "center of expansion" of the series. For instance, in the context of Taylor or power series, we usually have to change the center of expansion, that is, we have to consider a map $e_{n}$, for any $n \in \mathbb{N}$, from a set $L$ in $\mathbb{k}$ into a convenient set of functions, with $e_{n}(\xi)=(z-\xi)^{n}$. In the previous sections, we did not care about this, by considering two fixed families in $A$ and $X$, respectively $\left(e_{n}\right)_{n}$ and $\left(x_{n}\right)_{n}$, and we just considered expansions with respect to them. Moreover, most of the cases need working not only with a single space $X$, but with a countable union of such sets, for example when we work with entire functions that we approximate by universal series on different sets. This case is no more covered by our previous studies.

In [2], the authors extend their abstract theory of universal series to these "labeled universal series". They show that everything works in this latter context just as if we consider only one center of expansion and a single set $X$. Essentially, they give a generalization of Theorem 1.1 .

In this section, we will show that the existence of a closed infinitedimensional subspace consisting of universal series remains true in these two more general frameworks, and even when we combine them to take into account both the centers of expansions and the countable union of Fréchet spaces. The generalization of Theorem 1.1 is still at the heart of the proof, which is totally similar to the ones of Theorem 2.1 and Theorem 3.1, but more technical. In fact, the more extra parameters we will deal with, the 
more technical and heavier will be the proof. That is why we will just explain what we have to add to the previous proofs, and why this works.

4.1. The "center of expansion". We keep the notations $G$ and $X$ used previously. We will consider a set of universal series more general than the set $A \subseteq \mathbb{k}^{\mathbb{N}}$ in the first three sections, in order to work not only in a space of real or complex sequences, but directly in a convenient general Fréchet space on $\mathbb{k}$, where the notion of centers of expansion makes more sense. We denote by $\left(E, d_{E}\right)$ this space equipped with its complete translation-invariant metric and we suppose that its topology is given by a family of norms. Let also $L$ be a compact space, let $\xi_{0}$ be a fixed element of $L$, and suppose that, for any $n \in \mathbb{N}$, we have continuous maps

$$
e_{n}: L \rightarrow E, \quad x_{n}: L \rightarrow X \quad \text { and } \quad H_{n}: L \times E \rightarrow \mathbb{k}^{\mathbb{N}},
$$

with $H_{n}$ linear with respect to the second coordinate satisfying the condition

$$
H_{n}\left(\xi_{0}, e_{m}\left(\xi_{0}\right)\right)=\delta_{n, m} \quad \text { for every } n, m \in \mathbb{N} .
$$

The maps $e_{n}$ and $x_{n}$ generalize respectively the sequences $e_{n}$ in $A$ and $x_{n}$ in $X$ introduced in the first section.

Definition 4.1. For any $a \in G$, we denote by $g_{a}=\sum_{n=0}^{\infty} a_{n} e_{n}\left(\xi_{0}\right)$ the "polynomial" in $E$ associated to $a$. We define the valuation $v\left(g_{a}\right)$ and the degree $d\left(g_{a}\right)$ by $v\left(g_{a}\right)=v(a)$ and $d\left(g_{a}\right)=d(a)$, with the notations of the first section.

We make the following usual assumptions:

(1) The set $\left\{g_{a}: a \in G\right\}$ is dense in $E$.

(2) For every $a \in G$ and every $\xi \in L$, the sets $\left\{n: H_{n}\left(\xi, g_{a}\right) \neq 0\right\}$ are finite and uniformly bounded with respect to $\xi$.

(3) For every $a \in G$ and every $\xi \in L$,

$$
\sum_{n=0}^{\infty} H_{n}\left(\xi, g_{a}\right) e_{n}(\xi)=\sum_{n=0}^{\infty} a_{n} e_{n}\left(\xi_{0}\right)
$$

and the same is true in $X$ :

$$
\sum_{n=0}^{\infty} H_{n}\left(\xi, g_{a}\right) x_{n}(\xi)=\sum_{n=0}^{\infty} a_{n} x_{n}\left(\xi_{0}\right) .
$$

For example, we may take for $E$ the Fréchet space $H(D)$ of holomorphic functions on the disk, equipped with the standard metric of uniform convergence on compact sets, for $X$ the space $A(K)$ of holomorphic functions on $K$ continuous on $\bar{K}$, where $K$ is a compact set with connected complement and $K \cap D=\emptyset$, for $L$ a compact subset of $D$ and

$$
e_{n}(\xi)=x_{n}(\xi)=\left(z \mapsto(z-\xi)^{n}\right) \quad \text { and } \quad H_{n}(\xi, f)=\frac{f^{(n)}(\xi)}{n !}
$$


for $\xi \in D$ and $f \in H(D)$. The previous assumptions are clearly satisfied in this case.

Note that if $E=A$ and the maps $e_{n}$ (resp. $x_{n}$ ) are constant equal to $e_{n}\left(\xi_{0}\right)=e_{n} \in A$ (resp. to $x_{n} \in X$ ), and $H_{n}(\xi, a)=a_{n}$ for every $\xi \in L$ and $a=\left(a_{n}\right)_{n} \in A$, then we are precisely in the case covered by the previous sections.

Definition 4.2. We say that an element $f \in E$ is unrestrictively universal (in this framework) if, for every $x \in X$, there exists a sequence $\left(\lambda_{n}\right)_{n}$ of integers such that

$$
\sup _{\xi \in L} d_{X}\left(\sum_{n=0}^{\lambda_{N}} H_{n}(\xi, f) x_{n}(\xi), x\right) \underset{N \rightarrow \infty}{\longrightarrow} 0 .
$$

We denote by $U_{E, L}$ the set of all unrestricted universal series.

The generalized notion of restricted universal series is defined as follows:

Definition 4.3. We say that an element $f \in E$ is restrictively universal (in this framework) if, for every $x \in X$, there exists a sequence $\left(\lambda_{n}\right)_{n}$ of integers such that

$$
\begin{aligned}
& \sup _{\xi \in L} d_{E}\left(\sum_{n=0}^{\lambda_{N}} H_{n}(\xi, f) e_{n}(\xi), f\right) \underset{N \rightarrow \infty}{\longrightarrow} 0, \\
& \sup _{\xi \in L} d_{X}\left(\sum_{n=0}^{\lambda_{N}} H_{n}(\xi, f) x_{n}(\xi), x\right) \underset{N \rightarrow \infty}{\longrightarrow} 0 .
\end{aligned}
$$

We denote by $U_{E, L}^{R}$ the set of all restricted universal series.

Theorem 1.1 remains true in this context (see [2, Theorem 2, p. 8]):

THEOREM 4.1. Under the previous assumptions, the following are equivalent:

(1) $U_{E, L}^{R}$ is non-empty.

(2) For every $\varepsilon>0$, every $x \in X$, and every integer $p \geq 0$, there exists a polynomial $\left(a_{n}\right)_{n}$ with valuation $p$ such that

$$
\begin{aligned}
& \sup _{\xi \in L} d_{X}\left(\sum_{n \in \mathbb{N}} H_{n}\left(\xi, g_{a}\right) x_{n}(\xi), x\right)<\varepsilon, \\
& \sup _{\xi \in L} d_{E}\left(\sum_{n \in \mathbb{N}} H_{n}\left(\xi, g_{a}\right) e_{n}(\xi), 0\right)<\varepsilon .
\end{aligned}
$$

(3) For every $\varepsilon>0$, every $x \in X$, and every integer $p \geq 0$, there exists a polynomial $\left(a_{n}\right)_{n}$ with valuation $p$ such that

$$
d_{X}\left(\sum_{n \in \mathbb{N}} a_{n} x_{n}\left(\xi_{0}\right), x\right)<\varepsilon \quad \text { and } \quad d_{E}\left(\sum_{n \in \mathbb{N}} a_{n} e_{n}\left(\xi_{0}\right), 0\right)<\varepsilon .
$$

(4) $U_{E, L}^{R} \cup\{0\}$ contains a dense subspace of $E$. 
We are now able to give a generalization of Theorem 3.1 in the context of labeled universal series, taking into account the center of expansion:

THEOREM 4.2. With the previous assumptions, if $U_{E, L}^{R}$ is not empty, then $U_{E, L}$ contains a closed infinite-dimensional subspace of $E$, without 0 .

Idea of the proof. We keep the notations $\left(Q_{l}\right)_{l \in \mathbb{N}}$ and $\varphi, \psi$ introduced in Theorem 3.1 By proceeding exactly as in the proof of the latter, by considering $\left(e_{n}\left(\xi_{0}\right)\right)_{n}$ instead of $\left(e_{n}\right)_{n}$ (resp. $\left(x_{n}\left(\xi_{0}\right)\right)_{n}$ instead of $\left.\left(x_{n}\right)_{n}\right)$ and by the assumptions 4.2 and 4.3 , we construct three families $\left(g_{n, k}\right)_{n \geq k \geq 0}$, $\left(f_{n, k}\right)_{n \geq k \geq 0}$ and $\left(u_{n}\right)_{n \geq 0}$ of polynomials in $E$ (or $X$ with no confusion possible) with convenient valuation and degree as in the proof of Theorem 3.1 . such that

$$
\begin{aligned}
\sup _{\xi \in L}\left\|\sum_{j=0}^{\infty} H_{j}\left(\xi, g_{n, k}\right) x_{j}(\xi)-Q_{\varphi(n)}\right\|_{\psi(n)} & <\frac{1}{2^{N+4} K}, \\
\sup _{\xi \in L}\left\|\sum_{j=0}^{\infty} H_{j}\left(\xi, f_{n, k}\right) x_{j}(\xi)\right\|_{\psi(n)} & <\frac{1}{2^{n+4} K}, \\
d_{E}\left(f_{n+1, k}, f_{n, k}\right) & <\frac{1}{2^{n+4} K}, \\
\left\|\widehat{f} \widehat{f_{k, k}-u_{k}}\right\| & <\frac{1}{2^{n+3} K},
\end{aligned}
$$

and $\left(u_{n}\right)_{n \geq 0}$ being a basic sequence in $(\widehat{E}, \widehat{\|\cdot\|})$, the completion of $E$ for $\|\cdot\|$, one of the norms defining the topology of $E$. Then we construct a family $\left(f_{k}\right)_{k \geq 0}$ in $E$, using inequality (4.8), by

$$
f_{k}=\sum_{n \geq k}\left(f_{n+1, k}-f_{n, k}\right)+f_{k, k}
$$

for every $k \in \mathbb{N}$, and we define the closed subspace $F$ of $E$

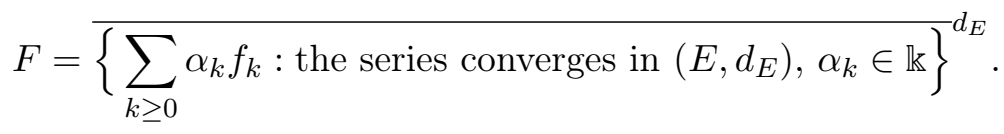

Then $F$ is infinite-dimensional because of the assumption (4.1) and the construction of the $f_{k}$ 's. The proof of the universality of every element of $F$ is quite similar to the one used for Theorem 3.1 and works after noticing that the "sup" is not an obstacle for the calculation. We do not go into details.

4.2. Universality in a countable family of Fréchet spaces. We keep the notations $G, E, L, e_{n}, H_{n}$ and $\xi_{0}$ of the previous section. In the proofs of Theorems 2.1, 3.1, or 4.2, the construction of the family $\left(f_{k}\right)_{k \geq 0}$, which is at the core of the proof, is such that certain parameters were taken 
into account. Namely, in the latter, the procedure was to approximate, for a countable family of norms, a countable family of polynomials simultaneously by some convenient countable families of other polynomials. For this, the strategy is to build a sequence of polynomials with multiple indices; each of these indices should correspond to an extra parameter through the functions $\varphi$ and $\psi$. More generally, this method allows us to add some extra "countable" parameters, making the formalism more complicated.

Therefore, we can extend Theorem 4.2 to the case of a countable family of Fréchet spaces $\left(\left(X_{n}, d_{n}\right)\right)_{n \geq 0}$ (where $d_{n}$ is the metric of $X_{n}$ ) instead of a single one $X$, and we can even work with a space $L$ which is not compact, but which is the union of an exhaustive sequence $\left(L_{m}\right)_{m}$ of compact subsets such that $\xi_{0} \in L_{0}$. Let also $\left(x_{n, k}\right)_{k \geq 0}$ be a sequence of continuous maps from $L$ to $X_{n}$, for any $n \in \mathbb{N}$. We shall modify the assumptions (1)-(3) given after Definition 4.1 as follows:

(1) The set $\left\{g_{a}: a \in G\right\}$ is dense in $E$.

(2) For every $a \in G$ and every $\xi \in L$, the sets $\left\{n: H_{n}\left(\xi, g_{a}\right) \neq 0\right\}$ are finite and uniformly bounded with respect to $\xi \in L_{m}$, for any $m \in \mathbb{N}$.

(3) For every $a \in G$ and every $\xi \in L$,

$$
\sum_{n=0}^{\infty} H_{n}\left(\xi, g_{a}\right) e_{n}(\xi)=\sum_{n=0}^{\infty} a_{n} e_{n}\left(\xi_{0}\right) .
$$

(4) For every $a \in G$, every $\xi \in L$ and every $n \geq 0$,

$$
\sum_{k=0}^{\infty} H_{k}\left(\xi, g_{a}\right) x_{n, k}(\xi)=\sum_{k=0}^{\infty} a_{k} x_{k}\left(\xi_{0}\right) .
$$

In this context, we redefine the notion of unrestricted universal series as follows:

DeFinition 4.4. We say that an element $f \in E$ is unrestrictively universal (in this framework) if, for every $n \in \mathbb{N}$ and every $x \in X_{n}$, there exists a sequence $\left(\lambda_{n}\right)_{n}$ of integers such that, for every $m \geq 0$,

$$
\sup _{\xi \in L_{m}} d_{X}\left(\sum_{k=0}^{\lambda_{N}} H_{k}(\xi, f) x_{n, k}(\xi), x\right) \underset{N \rightarrow \infty}{\longrightarrow} 0 .
$$

We denote by $U_{E, L}$ the set of all unrestricted universal series.

Next, we modify, the notion of restricted universal series:

Definition 4.5. We say that an element $f \in E$ is restrictively universal (in this framework) if, for every $n \in \mathbb{N}$ and every $x \in X_{n}$, there exists a 
sequence $\left(\lambda_{n}\right)_{n}$ of integers such that, for every $m \geq 0$,

$$
\begin{array}{r}
\sup _{\xi \in L_{m}} d_{E}\left(\sum_{k=0}^{\lambda_{N}} H_{k}(\xi, f) e_{k}(\xi), f\right) \underset{N \rightarrow \infty}{\longrightarrow} 0, \\
\sup _{\xi \in L_{m}} d_{X}\left(\sum_{n=0}^{\lambda_{N}} H_{k}(\xi, f) x_{n, k}(\xi), x\right) \underset{N \rightarrow \infty}{\longrightarrow} 0 .
\end{array}
$$

We denote by $U_{E, L}^{R}$ the set of all restricted universal series.

As Theorem 4.1 also extends to this framework ([2, Theorem 3]), it suffices to consider four functions $\varphi, \psi, \nu, \kappa$ from $\mathbb{N}$ to $\mathbb{N}$ such that, for every $(l, m, t, i) \in \mathbb{N}^{4}$, there exists an increasing sequence $\left(v_{k}\right)_{k}$ such that $\left(\varphi\left(v_{k}\right), \psi\left(v_{k}\right), \nu\left(v_{k}\right), \kappa\left(v_{k}\right)\right)=(l, m, t, i)$ for every $k$, and to follow the same proof as usual. Then, in this more general framework, we also have:

THEOREM 4.3. With the previous assumptions, if $U_{E, L}^{R}$ is non-empty, then $U_{E, L}$ contains a closed infinite-dimensional subspace of $E$ without 0.

5. Applications. This section is devoted to a short illustration of the previous results in some different frameworks, through classical examples of universality.

5.1. Applications in Banach spaces. We may first illustrate Theorem 2.1 by taking $A$ to be the Banach spaces $c_{0}$ or $l^{p}, 1 \leq p<\infty$; with their natural topologies, they are Banach subspaces of $\mathbb{C}^{\mathbb{N}}$ satisfying hypothesies (1)-(3) of Section 1, and the canonical sequence $\left(e_{n}\right)_{n}$ is basic. In [2], the authors apply their abstract theory to get the following:

Proposition 5.1. Let $A=c_{0}$ or $A=l^{p}, 1 \leq p<\infty$, endowed with their natural topologies. Then there exists a sequence $a=\left(a_{n}\right)_{n}$ in $A$ with the following property: For every compact set $K \subseteq \mathbb{C}$ with $K \cap \bar{D}=\emptyset$ and with connected complement, and for every function $h \in A(K)$, there exists a sequence $\left(\lambda_{n}\right)_{n}$ in $\mathbb{N}$ such that

$$
\left\|\sum_{j=0}^{\lambda_{n}} a_{j} z^{j}-h(z)\right\|_{\infty, K} \underset{n \rightarrow \infty}{\longrightarrow} 0
$$

where $\|\cdot\|_{\infty, K}$ is the supremum norm on $K$. Moreover, if we denote by $U_{A}$ the set of such sequences $a$, then $U_{A}$ is a dense $G_{\delta}$ subset of $A$, and it contains a dense vector subspace of $A$ without 0 .

Let us also notice that, combining results in Sections 1 and 4, it is clear that working with a Banach space $A$ on the one hand and a countable family of Fréchet spaces $\left(X_{n}, d_{n}\right)$ on the other hand yields the same conclusion as in Theorem 2.1 under the same usual assumptions. Then, with the previous 
notations, let $\left(X_{n}, d_{n}\right)$ be $\left(A\left(K_{n}\right),\|\cdot\|_{\infty, n}\right)$ where $A\left(K_{n}\right)$ is the set of continuous functions on $K_{n}$, holomorphic on $\stackrel{\circ}{K}_{n}$, and where $\left(K_{n}\right)_{n}$ is a family of compact sets of $\mathbb{C}$, with $K_{n} \cap \bar{D}=\emptyset$ and $K_{n}^{c}$ connected, and such that every compact set $K \subseteq \mathbb{C}$ with $K \cap \bar{D}=\emptyset$ and $K^{c}$ connected is contained in some $K_{n}$. Thanks to such a sequence $\left(K_{n}\right)_{n}$, it is clear that an application of Proposition 5.1 and of Theorem 4.3 in the present easier context gives the following result:

Proposition 5.2. With the notations of Proposition 5.1, the set $U_{A}$ contains a closed infinite-dimensional subspace of $A$ with 0 removed.

As in 2], we shall state a similar result in the context of Hardy or Bergman spaces, i.e. when $A=H^{p}(D)$ or $A=A^{p}(D), 1 \leq p<\infty$. Indeed, as before, Corollary 8 in [2] ensures that we have the following:

Proposition 5.3. Let $A=H^{p}(D)$ or $A=A^{p}(D), 1 \leq p<\infty$. Then there exists a closed infinite-dimensional subspace $F$ of $A$, without 0 , consisting of universal Taylor series, in the sense that for every $f=\sum_{n} a_{n} z^{n} \in F$, every compact set $K \subseteq \mathbb{C}$ with $K \cap \bar{D}=\emptyset$ and $K^{c}$ connected, and every $h \in A(K)$, there exists a sequence $\left(\lambda_{n}\right)_{n} \subseteq \mathbb{N}$ such that

$$
\left\|\sum_{n=0}^{\lambda_{n}} a_{n} z^{n}-h(z)\right\|_{\infty, K} \underset{n \rightarrow \infty}{\longrightarrow} 0,
$$

where $\|\cdot\|_{\infty, K}$ is the supremum norm on $K$.

A classical example of universal series is that of universal trigonometric series of Men'shov in the periodic case; see [11], 8] and 2].

The difference from our assumptions in Section 1 is that the topology of $X$ is not induced by a countable family of seminorms. $X$ is the space of complex measurable functions on $\mathbb{T}=\{z \in \mathbb{C}:|z|=1\}$ modulo the equivalence relation identifying two functions if they are identical off a subset with zero Lebesgue measure. If $f, g \in X$ then

$$
p_{X}(f, g)=\int_{\mathbb{T}} \frac{|f-g|}{1+|f-g|} d \lambda
$$

where $\lambda$ is the Lebesgue measure on $\mathbb{T}$. However, one can easily check that all our results hold in this case with $A=c_{0}$ or $A=l^{p}, p>2$. The universal series obtained in this way realize approximations in measure; but it turns out that these universal trigonometric series are identical with the universal trigonometric series realizing approximations in the almost everywhere sense. Thus we obtain the following:

Proposition 5.4. Let $A=c_{0}$ or $l^{p}, p>2$. There exists a sequence $a=$ $\left(a_{n}\right)_{n \geq 0} \in A$ such that, for every $2 \pi$-periodic complex measurable function $h$ : $\mathbb{R} \rightarrow \mathbb{C}$, there exists a sequence $\left(\lambda_{n}\right)_{n>1} \subseteq \mathbb{N}$ such that $\sum_{j=0}^{\lambda_{n}} a_{j} e^{i j x}$ converges 
to $h(x)$ almost everywhere as $n$ goes to infinity. The set of such sequences $a \in A$ contains a closed infinite-dimensional subspace of $A$ without 0 .

We can also have an analogous result on the torus $\mathbb{T}^{N}$ (see [2]).

Now, let $p_{0} \geq 0$. If for every $p>p_{0}$ there exists a universal series in $l^{p}$, then there exists a universal series in $\bigcap_{p>p_{0}} l^{p}$. This follows from the abstract theory ([2]) in combination with the definition of the topology in the Fréchet space $\bigcap_{p>p_{0}} l^{p}$ and is established in [15]. This fact, for $p_{0}=2$, in combination with the fact that there exists a universal trigonometric series in each $l^{p}$, $p>2$, implies the existence of a universal trigonometric series in $\bigcap_{p>2} l^{p}$. The result of Section 3 implies now that $\bigcap_{p>2} l^{p}$ contains a closed infinitedimensional subspace whose non-zero elements define universal trigonometric series of analytic type.

5.2. Applications in Fréchet spaces. In [9], the authors show the existence of universal series in $A=\bigcap_{p>1} l^{p}$ with respect to some sequences $\left(x_{n}\right)_{n}$ in abstract Fréchet spaces $X$, up to a special condition on $\left(x_{n}\right)_{n}$. More precisely, besides the usual assumptions made in the previous sections, they introduce the following condition, which they call (D):

Condition (D). For every finite set $I \subset \mathbb{N}$, there exist distinct indices $j_{n}(i), n \in \mathbb{N}, i \in I$, such that $x_{j_{n}(i)} \rightarrow x_{i}$ as $n \rightarrow \infty$.

We suppose that $A=\bigcap_{p>1} l^{p}$ is endowed with its usual topology of Fréchet space induced by a family of norms. The main abstract theorem they obtain is:

THEOREM 5.1. With the current notations, assume that $\left(x_{n}\right)_{n}$ satisfies Condition (D) and that the set of all finite linear combinations of $x_{n}$ 's is dense in $X$. Then $U_{\bigcap_{p>1} l^{p}} \neq \emptyset$. Moreover, $U_{\bigcap_{p>1} l^{p}}$ is a dense $G_{\delta}$ subset of $A=\bigcap_{p>1} l^{p}$ and it contains a dense vector subspace of $A$ without 0 .

Notice that the assumption that the set of all finite linear combinations of $x_{n}$ 's is dense in $X$ is not a restriction for us, since it is a consequence of the fact that $U_{A}$ is non-empty, which we have to assume in all our results in Sections 2 and 3. Theorem 5.1 just reveals that, in this context, we can replace the hypothesis $U_{A} \neq \emptyset$ by Condition (D) and $\overline{\operatorname{span}\left(x_{n}, n \in \mathbb{N}\right)}=X$. With this in mind, Theorems 5.1 and 4.3 yield this partially abstract result:

THEOREM 5.2. With the usual notations, set $A=\bigcap_{p>1} l^{p}$ and assume that $\left(x_{n}\right)_{n} \subseteq X$ satisfies Condition (D) and that $\overline{\operatorname{span}\left(x_{n}, n \in \mathbb{N}\right)}=X$. Then $U \cap A=U_{A}$ contains a closed infinite-dimensional subspace without 0 .

REMARK 5.3. We can easily see that Theorems 5.1 and 5.2 remain true if we replace $\bigcap_{p>1} l^{p}$ by any $l^{p}$ with $1<p<\infty$ or $c_{0}$. Therefore, it may be seen as a generalization of examples given in Section 5.1, except for the case 
$A=l^{1}$; indeed, the authors of [9] give an example where the statement of Theorem 5.1 does not hold when $A=l^{1}$.

We now give some "concrete" examples.

EXAMPLE 5.1. The simplest non-trivial couple $\left(X,\left(x_{n}\right)_{n}\right)$ satisfying the hypothesis of Theorem 5.2 is $\left(\mathbb{R},\left(x_{n}\right)=1\right)$. Indeed, $\left(x_{n}\right)_{n}$ satisfies Condition (D) and, of course, $\mathbb{R}=\operatorname{span}\left(x_{n}, n \in \mathbb{N}\right)$. In this setting, Theorem 5.1 first ensures that there exists a restricted universal sequence $a=\left(a_{n}\right)_{n} \in$ $A=\bigcap_{p>1} l^{p}(\mathbb{R})$ such that the set of all partial sums $\sum_{n=0}^{N} a_{n}$ is dense in $\mathbb{R}$ and, denoting by $U_{A}$ the set of all such sequences $a$, Theorem 5.2 yields the following result:

Proposition 5.5. With the previous notations, $U_{A}$ contains a closed infinite-dimensional subspace of $A$ without 0 .

EXAmple 5.2. A classical example of universal series is the one of universal trigonometric series. Set $X=\mathcal{C}(\mathbb{R})$, the set of all continuous complex functions on $\mathbb{R}$, equipped with the topology of uniform convergence on compact sets. Let us fix a sequence $\left(\beta_{n}\right)_{n} \subseteq \mathbb{R}$ without isolated points and define $x_{n}(t)=e^{i \beta_{n} t}$ for every $t \in \mathbb{R}$. As explained in [9, Section 5], $\left(x_{n}\right)_{n}$ satisfies Condition (D) and $X=\overline{\operatorname{span}\left(x_{n}, n \in \mathbb{N}\right)}$. Therefore, Theorems 5.1 and 5.2 give the following result:

THEOREM 5.4. With the previous notations, there exists a restricted universal sequence $a=\left(a_{n}\right)_{n} \in A=\bigcap_{p>1} l^{p}(\mathbb{C})$, i.e. such that for every continuous complex function $f$ on $\mathbb{R}$, there exists a sequence $\left(\lambda_{n}\right)_{n} \subseteq \mathbb{N}$ such that

$$
\sum_{n=0}^{\lambda_{n}} a_{n} e^{i \beta_{n} t} \underset{n \rightarrow \infty}{\longrightarrow} f(t) \quad \text { uniformly on every compact subset of } \mathbb{R} .
$$

Moreover, the set $U_{A}$ of all such sequences $a$ is a dense $G_{\delta}$ subset of $A$ and it contains a closed infinite-dimensional subspace without 0 .

For further applications, [9] provides a lot of examples which may illustrate Sections 2 and 3 of the present paper.

5.3. Universal series in simply connected domains. In [2], the authors gave a proof of the following theorem, using their abstract results on universality:

TheOREM 5.5. Let $\Omega$ be a simply connected domain in $\mathbb{C}$. There exists a holomorphic function $f$ on $\Omega$ such that its partial sums

$$
S_{N}(f, \xi)(z)=\sum_{n=0}^{N} \frac{f^{(n)}(\xi)}{n !}(z-\xi)^{n}, \quad \xi \in \Omega, N \in \mathbb{N},
$$

have the following property $(\mathrm{P})$ : 
For every compact set $K \subseteq \mathbb{C}$ with $K \cap \Omega=\emptyset$ and $K^{c}$ connected, and every function $h \in H(\mathbb{C})$, there exists a sequence $\left(\lambda_{n}\right)_{n}$ of integers such that, for every compact set $L \subseteq \Omega$ and every $l \in \mathbb{N}$, we have:

(1) $\sup \sup \left|S_{\lambda_{n}}(f, \xi)(z)-f(z)\right| \rightarrow 0$ as $n \rightarrow \infty$, $\xi \in L, z \in L$

(2) $\sup _{\xi \in L} \sup _{z \in K}\left|\frac{d^{l}}{d z^{l}}\left(S_{\lambda_{n}}(f, \xi)\right)(z)-h^{(l)}(z)\right| \rightarrow 0$ as $n \rightarrow \infty$.

With the notations of the fourth section, set $E=H(\Omega)$, endowed with the topology of uniform convergence on compacta, and let $d$ be the translation-invariant metric on $E$ defined by the family of seminorms $\|f\|_{n}=$ $\sup _{z \in L_{n}}|f(z)|$, where $f \in E$ and $\left(L_{n}\right)_{n}$ is an exhausting family of compact sets of $\Omega=\bigcup_{n=0}^{\infty} L_{n}$. Let also $X_{n}, n \geq 0$, denote $H(\mathbb{C})$ equipped with the metric $d_{n}$ induced by the family of seminorms $\sup _{z \in K_{n}}\left|f^{(l)(z)}\right|, l \geq 0$, where $K_{n}$ is a family of compact sets of $\mathbb{C}$ with $K_{n} \cap \Omega=\emptyset$ and $K_{n}^{c}$ connected, and such that every compact set $K \subseteq \mathbb{C}$ with $K \cap \Omega=\emptyset$ and $K^{c}$ connected is contained in some $K_{n}$ (see [2, B-2] for the existence of such a sequence). Moreover, for $\xi \in \Omega, f \in E$ and $k \geq 0$, define $e_{k}(\xi)=\left(z \mapsto(z-\xi)^{k}\right) \in E$, $x_{n, k}=\left(z \mapsto(z-\xi)^{k}\right) \in X_{n}$ and $H_{k}(\xi, f)=f^{(k)}(\xi) / k ! \in \mathbb{C}$. As explained in [2], all the assumptions of the fourth section are satisfied and the previous theorem ensures that $U_{E, L} \neq \emptyset$ with $L=\Omega$. Hence, Theorem 4.3 gives:

TheOREM 5.6. Let $\Omega$ be a simply connected domain in $\mathbb{C}$. There exists a closed infinite-dimensional subspace of $(E, d)=(H(\Omega), d)$ consisting of holomorphic functions such that all their partial sums (apart from 0) have the property $(\mathrm{P})$ of Theorem 5.5 .

Since universal Taylor series of the previous type in simply connected domains have Ostrowski's gaps ([13]), condition (P) of Theorem 5.5 is equivalent to the corresponding condition for only one center. Thus, the result of Section 3 suffices for the proof of Theorem 5.6. This is not the case if we assume that the universal approximation is valid on part of the boundary, that the universal function is smooth on another part of the boundary and that the supremum with respect to $\zeta$ in (2) is taken on some sets $L$ which may contain parts of the boundary ([2]). Then a result analogue to Theorem 5.6 holds.

5.4. Fekete's theorem. We finish the illustration of our abstract study by an example which shows one of the limits of Theorem 2.1 or Theorem 3.1. It may appear a little ironical as it is known to be at the beginning of universal series study. Indeed, as far as we know, the first universal series was given by Fekete in 1914 (see [16]): 
THEOREM 5.7 (Fekete's theorem (1914)). There exists a real sequence $\left(a_{n}\right)_{n \geq 0}$ such that, for any continuous real function $f$ on $[-1,1]$ satisfying $f(0)=0$, there exists a sequence of integers $\left(\lambda_{n}\right)_{n \geq 0}$ such that

$$
\sum_{n=0}^{\lambda_{N}} a_{n} x^{n} \underset{N \rightarrow \infty}{\longrightarrow} f \quad \text { uniformly on }[-1,1] \text {. }
$$

With the notations of the second section, we set $A=\mathbb{R}^{\mathbb{N}}$ with the Cartesian topology; let also $X$ be the space of all continuous real functions on $[-1,1]$ vanishing at 0 , with the topology of uniform convergence. Fekete's theorem ensures that $U_{A}$ is not empty, and Theorem 1.1 applies and gives the following result:

TheOREm 5.8. Denote by $U_{A}$ the set of all sequences $\left(a_{n}\right)_{n \geq 0}$ such that, for every continuous real function $f$ on $[-1,1]$ vanishing at 0 , there exists a sequence $\left(\lambda_{n}\right)_{n>0}$ of integers such that

$$
\sum_{n=1}^{\lambda_{N}} a_{n} x^{n} \underset{N \rightarrow \infty}{\longrightarrow} f \quad \text { uniformly on }[-1,1] .
$$

Then $U_{A}$ is a dense $G_{\delta}$ subset of $A$ and it contains a dense vector subspace of $A$ without 0 .

Although this context is covered by Theorem 1.1, it is not the case of our Theorem 3.1. for technical reasons, the latter requires the existence of a continuous norm on $A$ (condition (3.1)) and it is well-known that there is no continuous norm on $\mathbb{K}^{\mathbb{N}}$. As we said at the beginning of Section 3 , the authors of [4] show that if (3.1) is removed, then the existence of a closed infinite-dimensional subspace of universal vectors in similar contexts fails. We can legitimately wonder if in Fekete's context there does not exist a closed infinite-dimensional subspace with 0 removed consisting of universal series. The next result positively answers this question:

TheOREM 5.9. In Fekete's context, there does not exist a closed infinitedimensional subspace without 0 consisting of universal series.

The proof is essentially based on the following general lemma:

Lemma 5.1. Let $F$ be an infinite-dimensional subspace of $\mathbb{R}^{\mathbb{N}}$. Denote by $\left(e_{n}\right)_{n}$ the canonical sequence in $\mathbb{R}^{\mathbb{N}}$; as in Section $1, v(u)$ is the valuation of $u \in \mathbb{R}^{\mathbb{N}}$, with respect to $\left(e_{n}\right)_{n}$. Then there exists a sequence $\left(u_{n}\right)_{n}$ in $F-\{0\}$ such that $\left(v\left(u_{n}\right)\right)_{n}$ is increasing.

Proof of Lemma 5.1. The construction of $\left(u_{n}\right)_{n}$ uses the following observation:

Claim. Let $\left(u_{0}, \ldots, u_{n}\right)$ be a finite family of non-zero elements of $F$ such that

$$
v\left(u_{0}\right)<v\left(u_{1}\right)<\cdots<v\left(u_{n}\right)
$$


and if $u \in F-\{0\}$ satisfies $v(u) \leq v\left(u_{n}\right)$, then $v(u)=v\left(u_{i}\right)$ for some $i$, $0 \leq i \leq n$. Then there exists $w \in F$ such that $v(w)>v\left(u_{n}\right)$.

Proof of the Claim. Denote

$$
E_{n}=\left\{w \in F: v(w)>v\left(u_{n}\right)\right\} .
$$

By contradiction, suppose that $E_{n}=\{0\}$. Because of (5.1), if $w \in F-\{0\}$ then there exist real $\lambda_{0}, \ldots, \lambda_{n}$ such that one of the following two cases holds:

(1) $w-\left(\lambda_{0} u_{0}+\cdots+\lambda_{n} u_{n}\right)=0$ or

(2) $v\left(w-\left(\lambda_{0} u_{0}+\cdots+\lambda_{n} u_{n}\right)\right)>v\left(u_{n}\right)$.

As the second case is impossible since we supposed that $E_{n}=\{0\}$, it follows that $w$ is a finite linear combination of $u_{i}$ 's, $0 \leq i \leq n$. This contradicts the fact that $F$ is infinite-dimensional.

We now finish the proof of Lemma 5.1. We construct a sequence $\left(u_{n}\right)_{n}$ of non-zero elements of $F$ such that, for any integer $n$, the sequence $\left(u_{0}, \ldots, u_{n}\right)$ satisfies the assumptions of the claim. We define $u_{0}$ to be a non-zero element of $F$ with minimal valuation. Next, suppose that $\left(u_{0}, \ldots, u_{n}\right)$ has been built and apply the Claim to this family. We know that there exists $w \in F$ such that $v(w)>v\left(u_{n}\right)$. We define $u_{n+1}$ to be such an element with $v\left(u_{n+1}\right)$ minimal. It remains to show that $\left(u_{0}, \ldots, u_{n+1}\right)$ still satisfies the assumption of the Claim. Pick $u \in F$ with $v(u) \leq v\left(u_{n+1}\right)$. If $v(u) \leq v\left(u_{n}\right)$, then we may apply the induction hypothesis. Otherwise, $v(u)=v\left(u_{n+1}\right)$ by minimality of $v\left(u_{n+1}\right)$. The sequence $\left(u_{n}\right)_{n}$ which we construct following this process clearly satisfies the conclusion of Lemma 5.1.

We turn to the proof of Theorem 5.9 .

Proof of Theorem 5.9. Let $\left(u_{n}\right)_{n}$ be a sequence as in Lemma 5.1. Because

$$
v\left(u_{n}\right)<v\left(u_{n+1}\right) \quad \text { for every } n \in \mathbb{N},
$$

we observe that for any real sequence $\left(\alpha_{n}\right)_{n}, \sum_{n=0}^{\infty} \alpha_{n} u_{n}$ is convergent in $\mathbb{R}^{\mathbb{N}}$. Now, in the notation of Section 2 , we can find a sequence $\left(\alpha_{n}\right)_{n} \in \mathbb{R}^{\mathbb{N}}$ such that, for any $n \geq 0$,

$$
\left\|S_{p}\left(\alpha_{0} u_{0}+\cdots+\alpha_{n} u_{n}, X\right)\right\|_{\infty}>1 \quad \text { for } p \in\left[v\left(u_{n}\right), v\left(u_{n+1}\right)[.\right.
$$

Indeed, we may choose $\alpha_{0} \in \mathbb{R}$ such that

$$
\left\|S_{p}\left(\alpha_{0} u_{0}, X\right)\right\|_{\infty}>1
$$

for any $p \in\left[v\left(u_{0}\right), v\left(u_{1}\right)\left[\right.\right.$, because $S_{p}\left(u_{0}, X\right) \neq 0$ for any $p$ in this interval. If $\alpha_{0}, \ldots, \alpha_{n}$ have been built, we construct $\alpha_{n+1}$ as follows. We observe that

$$
\begin{aligned}
\| S_{p}\left(\alpha_{0} u_{0}+\cdots+\right. & \left.\alpha_{n} u_{n}+\alpha_{n+1} u_{n+1}, X\right) \|_{\infty} \\
& =\left|\alpha_{n+1}\right|\left\|S_{p}\left(\frac{\alpha_{0}}{\alpha_{n+1}} u_{0}+\cdots+\frac{\alpha_{n}}{\alpha_{n+1}} u_{n}+u_{n+1}, X\right)\right\|_{\infty},
\end{aligned}
$$


and since $S_{p}\left(u_{n+1}, X\right) \neq 0$ for any $p \in\left[v\left(u_{n+1}\right), v\left(u_{n+2}\right)\right.$ [, we can choose $\left|\alpha_{n+1}\right|$ sufficiently large in order to have (5.2). Now, by construction, it follows that for every $p \in \mathbb{N}$, there exists $n \in \mathbb{N}$ such that $p \in\left[v\left(u_{n}\right), v\left(u_{n+1}\right)[\right.$ and

$$
\left\|S_{p}\left(\sum_{k=0}^{\infty} \alpha_{k} u_{k}, X\right)\right\|_{\infty}=\left\|S_{p}\left(\sum_{k=0}^{n} \alpha_{k} u_{k}, X\right)\right\|_{\infty}>1 .
$$

This means that every partial sum of $\sum_{n=0}^{\infty} \alpha_{n} u_{n}$ in $X$ has supremum norm greater than 1 , hence no continuous function with range in ]-1, 1[ can be approximated by subsequences of partial sums of $h:=\sum_{n=0}^{\infty} \alpha_{n} u_{n}$. Therefore $h$ fails to be universal in Fekete's sense; now, as $F$ has been supposed to be closed, $h \in F$ and the proof is complete.

Acknowledgments. I would like to thank the referee for carefully reading this paper and giving many valuable comments and references.

\section{References}

[1] F. Bayart, Linearity of sets of strange functions, Michigan Math. J. 53 (2005), 291-303.

[2] F. Bayart, K.-G. Grosse-Erdmann, V. Nestoridis and C. Papadimitropoulos, Abstract theory of universal series and applications, Proc. London Math. Soc. 96 (2008), 417-463.

[3] F. Bayart and É. Matheron, Dynamics of Linear Operators, Cambridge Tracts in Math. 179, Cambridge Univ. Press, 2009.

[4] J. Bonet, F. Martínez-Giménez and A. Peris, Universal and chaotic multipliers on spaces of operators, J. Math. Anal. Appl. 297 (2004), 599-611.

[5] K.-G. Grosse-Erdmann, Holomorphe Monster und universelle Funktionen, Mitt. Math. Sem. Giessen 176 (1987).

[6] -, Universal families and hypercyclic operators, Bull. Amer. Math. Soc. 36 (1999), $345-381$.

[7] J.-P. Kahane, Baire's category theorem and trigonometric series, J. Anal. Math. 80 (2000), 143-182.

[8] J.-P. Kahane and V. Nestoridis, Séries de Taylor et séries trigonométriques universelles au sens de Menchoff, J. Math. Pures Appl. 79 (2000), 855-862.

[9] S. Koumandos, V. Nestoridis, Y.-S. Smyrlis and V. Stefanopoulos, Universal series in $\bigcap_{p>1} l^{p}$, Bull. London Math. Soc., to appear.

[10] J. Lindenstrauss and L. Tzafriri, Classical Banach Spaces I, Springer, 1977.

[11] D. Menchoff [D. Men'shov], Sur les séries trigonométriques universelles, C.R. (Doklady) Acad. Sci. URSS (N.S.) 49 (1945), 79-82.

[12] A. Montes-Rodríguez, Banach spaces of hypercyclic vectors, Michigan Math. J. 43 (1996), 419-436.

[13] J. Müller, V. Vlachou and A. Yavrian, Universal overconvergence and Ostrowskigaps, Bull. London Math. Soc. 38 (2006), 597-606.

[14] V. Nestoridis and C. Papadimitropoulos, Abstract theory of universal series and an application to Dirichlet series, C. R. Math. Acad. Sci. Paris 341 (2005), 539-543. 
[15] V. Nestoridis and V. Stefanopoulos, Universal series and approximate identities, submitted.

[16] J. Pál, Zwei kleine Bemerkungen, Tôhoku Math. J. 6 (1915), 42-43.

Stéphane Charpentier

Institut de Mathématiques de Bordeaux

351 Cours de la Libération

33405 Talence Cedex, France

E-mail: stephane.charpentier@math.u-bordeaux1.fr

Received May 18, 2009

Revised version March 25, 2010 